\title{
Los tiempos cambian, la Revista cambia
}

La visibilidad y la divulgación del conocimiento científico y tecnológico se han venido potenciando a través de las plataformas informáticas que permiten una interacción humana nunca antes vista, que trasciende las diferencias étnicas, culturales, geográficas e idiomáticas. Gracias a ello estamos viviendo en un mundo globalizado donde pueden observarse simultáneamente todos los procesos del mundo científico, especialmente por medio de las publicaciones que, como conducto de una interacción constructiva, fácil y rápida entre pares científicos, permiten la comunicación de los saberes potenciadora del avance del conocimiento. Los tiempos cambian y nosotros, inevitablemente, somos parte de ese cambio. No podemos llegar tarde a este gran diálogo científico global.

La revista de la Academia Colombiana de Ciencias Exactas, Físicas y Naturales empezó su existencia el 1 de diciembre de 1936, poco tiempo después de su legalización por parte del Gobierno de Colombia a través del decreto 1218 de ese mismo año. Después de 78 años de publicación hemos llegado ya al número 146 del volumen 38. Como puede verse en este número, el equipo editorial de la revista ha cambiado y con él, también el proceso editorial, lo cual es de especial interés para los autores y para la audiencia lectora, pues se busca garantizar que cada artículo publicado reciba una adecuada evaluación por parte de pares de reconocimiento nacional e internacional y que el tiempo entre el sometimiento y la eventual publicación de los manuscritos se reduzca al mínimo. Además, la circulación del conocimiento y la necesidad de construir referencias estructuradas sobre las publicaciones en dominios particulares de la ciencia y la tecnología, así como de conocer lo que se produce y acceder a los documentos de las publicaciones, nos obligan a utilizar las mejores bases de datos especializadas en la indexación de revistas científicas.

En este sentido, considero de la mayor importancia dar a conocer aquí a nuestros lectores y contribuyentes, los integrantes del equipo editorial que nos acompañará a partir del número 146. Doy entonces la bienvenida a la doctora Elizabeth Castañeda, a quien tendré el gusto de acompañar en la tarea de editora en jefe, y a todos y cada uno de los miembros del Comité Editorial: Bernardo Gómez, Pedro Fernández, Rubén Antonio Vargas, Víctor Albis, Gabriel Roldán, Gonzalo Andrade, Jaime Cantera, José Luis Fernández, Thomas Defler, German Poveda, Armando Espinosa, Carlos Alberto Vargas, José Ignacio Martínez, Guillermo Páramo, Rubén Ardila, Felipe Guhl, Luis Fernando García, Fanor Mondragón, Moisés Wasserman y Sonia Moreno y a los miembros del Comité Científico Internacional: José Rubens Pirani, del Departamento de Botánica, Instituto de Biociencias, Universidad de Sao Paulo, Brasil; Gioconda San Blas, de la Oficina de Ciencia y Tecnología del Gobierno de Miranda, Venezuela, y Primera Vicepresidenta de la Academia de Ciencias Físicas, Matemáticas y Naturales de Venezuela; Carlos Jiménez, de la Facultad de Ciencias, Departamento de Química Fundamental, Universidad de La Coruña, España; Antoine M. Cleef, del Institute for Biodiversity and Ecosystem Dynamics (BED), Faculty of Science, University of Amsterdam, Science Park, The Netherlands; Wolfgang Gieren, del Departamento de Astronomía, Universidad de Concepción, Chile; Ángela Guzmán, de la University of Central Florida, Orlando, EEUU, seleccionados por su producción científica y por su experiencia en el mundo de las publicaciones en el área de las Ciencias básicas. Estamos seguros que con el concurso de todos, cumpliremos con los objetivos que nos hemos trazado para los próximos años.

Por otro lado, es importante resaltar que la importancia de una revista científica constituye un criterio determinante para los autores en el momento de decidir dónde publicar. Está ampliamente aceptado en el seno de la comunidad científica que dicha importancia se puede determinar por la evaluación que de ella hagan los sistemas de indexación, particularmente aquellos que tienen mayor aceptación en el ámbito internacional, ya que desempeñan un papel orientador en la difusión de las publicaciones seriadas que cumplen con los requisitos científicos y editoriales, establecidos por las entidades responsables de estos servicios.

En la actualidad, la revista está reseñada en varios índices internacionales tales como Actualidad Iberoamericana, Agris, CIRS (International Center for Scientific Research), Current Mathematical Publications, EBS-CO, Dialnet, Historia Mathematica, Index Kewensis, Latindex, Mathematical Reviews, MathSciNet, PERIODICA, SciELO, Online Computer Library CenterOCLC, Zentralblatt für Mathematik, Zoological Record, RLG, y en Colombia se encuentra en la base de datos Publindex. Los planes para el inmediato futuro comprenden la indexación de la revista en ISI/SCOPUS, consideradas las bases de datos de revistas científicas con mayor índice de citación en el ámbito internacional. 
Por otra parte, con el fin de mejorar la gestión de edición hemos seleccionado al Open Journal System (OJS), un software de código abierto para la gestión en línea de revistas científicas, el cual ha sido desarrollado y es continuamente actualizado por el Public Knowledge Project (PKP) (una iniciativa de varias universidades americanas a través de la cual se desarrolló el software mencionado con el objetivo de mejorar la calidad del proceso de publicación y el acceso a las publicaciones científicas). El OJS agiliza la comunicación entre los contribuyentes, el equipo editorial y los lectores en cada uno de los pasos del proceso, desde el sometimiento de los artículos hasta su publicación en línea y su indexación, es decir, el envío y la recepción de artículos y contribuciones en línea, la selección de los editores, la elección de revisores, el establecimiento de recordatorios automáticos, el manejo de fechas límite para la entrega de correcciones, la decisión final de aceptación por parte de los editores, el envío de los documentos al corrector de estilo y al diagramador, hasta llegar finalmente a la publicación electrónica de los artículos y su transferencia para la indexación en las bases de datos y los buscadores. Actualmente, el PKP es utilizado por cerca de 15.000 revistas científicas en todo el mundo (htttp://www.pkp.sfu.ca, 31 de mayo de 2014).

Además, la apariencia externa de la revista también está teniendo cambios. La carátula, por ejemplo, mostrará anualmente los símbolos de la Academia o aquellos relacionados con las diferentes secciones de la revista y su contenido. Para el número 146 hemos seleccionado la especie de Mutisia que aparece en la parte superior derecha del escudo de la Academia. Esta planta fue dedicada por Linneo a José Celestino Mutis, quien fue el primer gran promotor de la actividad científica institucional en Colombia durante la segunda mitad del siglo XVIII. De igual forma, la contraportada, la página legal y las portadillas de los artículos se han ajustado a las exigencias del ISI/SCOPUS. Igualmente, en algunas ocasiones solicitaremos editoriales a científicos de alto reconocimiento para exaltar temas de investigación que se publiquen en la revista. Por último es importante mencionar que en los próximos meses haremos el tránsito de la revista también a versión electrónica.

Naturalmente, no podemos concluir este editorial sin invitar a nuestros contribuyentes y autores, así como a todos los editores y personal que trabaja con nosotros a construir este nuevo camino hacia la modernización de ésta importante revista científica.

\section{Carmenza Duque}

Editora en Jefe 